\title{
Intradermal Injection of Normal Saline for Treatment of Fat Atrophy Following Corticosteroid Injection
}

\author{
Sierra Crowe, MD ${ }^{1}$ E. Chad Schmidgal, $\mathrm{MD}^{2}$
}

A 20-year-old woman with a Fitzpatrick score of 2 presented via telehealth with "divots" on her forehead and scalp. She reported that 2 months prior to presentation she had received multiple steroid injections into her forehead and posterior skull by a neurologist for treatment of migraines. She had soon thereafter developed fat atrophy in 3 areas: 2 on her forehead and 1 on her occipital scalp. Although they were not painful or otherwise symptomatic, she was unhappy with the cosmetic appearance. She had no other skin or systemic symptoms.

She visited the office the following day, and on examination, 2 atrophic plaques were noted on her bilateral forehead around the mid-pupillary lines, with a larger atrophic plaque on the occipital scalp (Figures 1 and 2).

Given the location and timing of the lesions, they were consistent with skin atrophy induced by her prior steroid injections. Therefore, a biopsy was not performed.

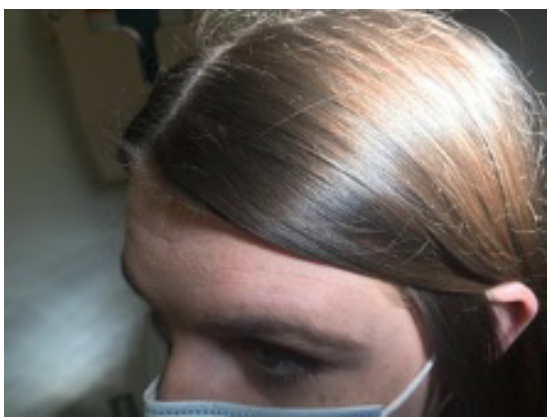

Figure 1. Left forehead plaque prior to treatment

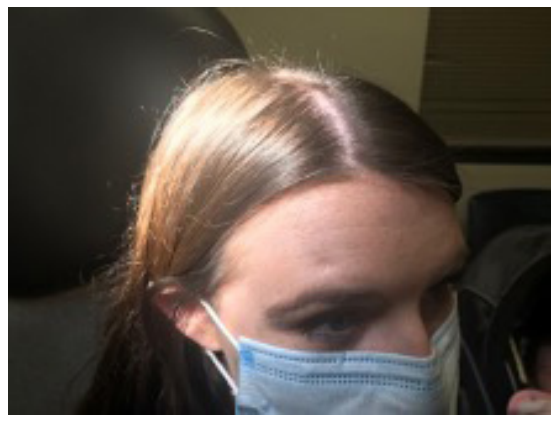

Figure 2. Right forehead plaque prior to treatment

\section{AFFILIATIONS:}

${ }^{1}$ Flight Surgeon, Naval Air Station Lemoore, Lemoore, California

${ }^{2}$ Dermatologist, Naval Medical Center San Diego, San Diego, California

\section{CITATION:}

Crowe S, Schmidgal CE. Intradermal injection of normal saline for treatment of fat atrophy following corticosteroid injection. Consultant. Published online February 25, 2022. doi:10.25270/con.2022.02.00008

Received September 22, 2021. Accepted October 1, 2021.

\section{DISCLOSURES:}

The authors report no relevant financial relationships.

\section{DISCLAIMER:}

The views expressed in this article are those of the authors and do not necessarily reflect the official policy or position of the Department of the Navy, the Department of Defense, or the US Government.

\section{CORRESPONDENCE:}

Sierra Crowe, MD, Naval Air Station Lemoore, 1215 North Taylor Avenue, Hanford, CA 93230 (crowesierra@gmail.com)

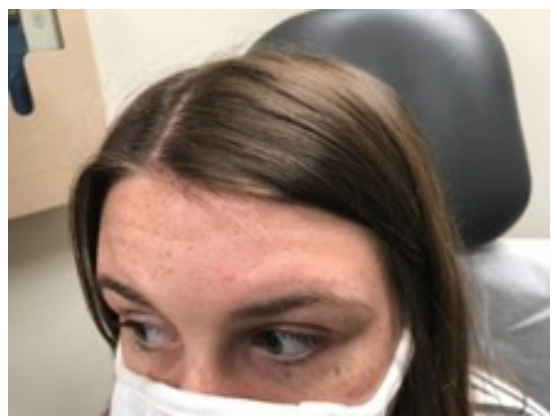

Figure 3. Near complete resolution of left forehead plaque after one month of treatment

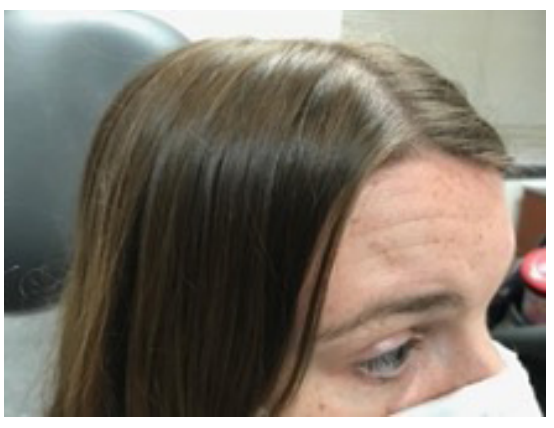

Figure 4. Significant improvement of right forehead plaque after one month of treament

\section{Treatment}

She was counseled regarding different treatment options: no intervention, serial saline injections, or referral to plastic surgery for consideration of fat grafting or filler placement. Our patient elected to undergo serial saline injections. These injections were repeated weekly for 6 weeks with complete resolution of the left forehead plaque (Figure 3) and significant improvement of the right forehead plaque (Figure 4) and occipital scalp lesions.

\section{Discussion}

Corticosteroid injections are a common 
therapy that can infrequently cause adverse effects, such as fat atrophy. The injections are used to treat several conditions, particularly dermatologic, musculoskeletal, and allergic conditions including arthritis, bursitis, tendinitis, alopecia, psoriasis, keloids and hypertrophic scars, and severe allergies. Although these injections are considered low risk, they can cause well-known adverse effects, such as tendon weakening or rupture, infection, bleeding, skin discoloration, skin and fat atrophy, and damage to cartilage and bone. ${ }^{1}$ The incidence of fat atrophy is not well known but has been estimated to be between $3 \%$ and $41 \%$. 'This adverse effect is more frequent with intramuscular injections, such as those used to treat allergies; however, fat atrophy has been reported in intra-articular and peritendinous injections, as well as with oral corticosteroids. ${ }^{3,4}$ These risks are increased with larger or multiple doses and with corticosteroids of high concentration or low solubility. ${ }^{5}$ For unclear reasons, corticosteroid-induced skin and fat atrophy appears to occur more frequently in premenopausal women than in men. ${ }^{2,3}$

Fat atrophy that results from corticosteroid use typically self-resolves in 6 to 12 months. ${ }^{5}$ After this time, if the atrophy persists, surgical intervention such as fat grafting or injection is often performed if the areas are bothersome to the patient. Because fat atrophy is known to resolve spontaneously, acute treatment is often not pursued. However, these lesions can be significant, located in cosmetic areas, and problematic for the patient, which are all reasons why acute treatment may be pursued. Several case reports demonstrate the efficacy of normal saline injections for treatment of corticosteroid-induced fat atrophy. ${ }^{6-8}$ In this case report, we provide further evidence for an infrequently considered treatment method, as well as methods to decrease the risk of atrophy with corticosteroid use.

Our case demonstrates the utility of normal saline injections for corticosteroid-induced fat atrophy. Although fat atrophy may resolve spontaneously, these lesions may be unsightly and undesirable to the patient, who may wish for them to be reversed more quickly. In this case, our patient was a young woman with 2 of the atrophic plaques in a cosmetically sensitive area. Lesions on the facial area can be distracting and obvious, even when relatively small. These plaques were not only significant in size, but they were also in an area that was difficult to camouflage with hair or hats; makeup is poorly effective in disguising blemishes of such depth.

After injection, corticosteroid is deposited in the area of treatment with differing levels of solubility depending on the formulation. Corticosteroid crystals can persist in the subcutaneous tissue for months to years when less-soluble formulations are used, occasionally causing fat involution. The exact mechanism of action is poorly understood, but studies suggest that corticosteroid crystals persist in the subcutaneous tissue, resulting in fat involution. ${ }^{2}$ Histopathology typically demonstrates smaller and fewer fat cells, as well as lipophage-like macrophages without evidence of inflammation or necrosis. ${ }^{3,9}$ Glucocorticoids also reduce the production of type 1 and type 3 collagen.10

Over time, these effects will gradually dissipate as the body reabsorbs the corticosteroid crystals and the adipocytes return to normal number and size. Usually, this will be evident within the first year, but sometimes the atrophy is permanent. ${ }^{5}$ In these situations, if correction is desired, then other procedural techniques are required to restore normal skin topography, such as filler placement or fat grafting.

Because our patient was within the 1-year timeframe, saline injections were performed. Saline injections aid in diluting the corticosteroid that remains in the skin, facilitating a more rapid reversal of fat atrophy than the patient would otherwise experience. It has been proposed that normal saline injections resuspend the corticosteroid crystals, allowing the body's natural mechanisms to identify and remove the foreign bodies; however, further studies are needed to determine the precise mechanism of action. ${ }^{11}$ Case reports have demonstrated resolution in 4 to 8 weeks with normal saline injections. ${ }^{7}$ Autologous fat transfer was less favored as a treatment option, as our patient still had a high likelihood of natural reversal of atrophy. Injecting the area with filler or fat could have led to eventual overcorrection, as the remaining corticosteroid was gradually removed from the area and the patient's natural fat architecture returned. Additionally, the forehead presents a difficult area to correct with such procedures, as there is very little subcutaneous tissue to allow for blending or to help camouflage the area. Therefore, scarring is more obvious, making these interventions more apparent.

Although we could find no published comparative studies, utilizing certain techniques may decrease the chance of fat atrophy based on our current understanding of the pathophysiology of this condition. For deeper structures, an insoluble formulation such as triamcinolone acetonide or hexacetonide may be used. For more superficial injections such as tendons and skin lesions, more soluble compounds such as betamethasone sodium phosphate and acetate or dexamethasone sodium phosphate could be considered. ${ }^{3,12}$ Care should be taken to ensure as much of the injection reaches the intended site, while minimizing the chance of spread. ${ }^{2}$ This can be optimized by choosing a properly sized needle and completing the injection in one push. Multiple injections or injecting while withdrawing the needle should be avoided. Intramuscular injections should be injected at an appropriate depth to reach the muscle, typically 1.5 to 2 inches but varies with body habitus, to avoid injecting corticosteroid into adipose tissue. ${ }^{5}$ In addition, the clinician should visibly inspect the medication before and during injections to ensure it has not precipitated.

\section{Conclusions}

Medical practitioners should be aware of the less common adverse effects of steroid injections, such as fat atrophy. A 
history of corticosteroid injections should be elucidated if a patient presents with fat atrophy of unknown cause, as this can prevent misdiagnosis and an otherwise lengthy, invasive, and expensive medical workup for other causes. When performing corticosteroid injections, care should be taken to minimize the risk of fat atrophy. Although fat atrophy is generally not dangerous and frequently resolves on its own, it can present in cosmetically sensitive areas requiring more rapid reversal. For these cases, serial normal saline injections can provide a simple, safe, and effective method to permanently reverse corticosteroid-induced fat atrophy.

\section{References}

1. Brinks A, Koes BW, Volkers AC, Verhaar JA, Bierma-Zeinstra SM. Adverse effects of extra-articular corticosteroid injections: a systematic review. BMC Musculoskelet Disord. 2010;11:206. doi:10.1186/1471-2474-11206

2. Dahl PR, Zalla MJ, Winkelmann RK. Localized involutional lipoatrophy: a clinicopathologic study of 16 patients. J Am Acad
Dermatol. 1996;35(4):523-528. doi:10.1016/ s0190-9622(96)90673-8

3. Jacobs MB. Local subcutaneous atrophy after corticosteroid injection. Postgrad Med. 1986;80(4):159-160. doi:10.1080/00325481.19 86.11699543

4. Khoo A, Grattan CE. Making a dent with corticosteroid injections for de Quervain's tenosynovitis. BMJ Case Rep. 2016;2016:bcr2015214225. https://doi. org/10.1136/bcr-2015-214225

5. Park SK, Choi YS, Kim HJ. Hypopigmentation and subcutaneous fat, muscle atrophy after local corticosteroid injection. Korean J Anesthesiol. 2013;65(6 Suppl):S59-S61. doi:10.4097/kjae.2013.65.6S.S59

6. Margulies SL, Morris A. Successful treatment of lipoatrophy with normal saline. JAAD Case Rep. 2015;1(6):415-417. doi:10.1016/j.jdcr.2015.10.008

7. Shumaker PR, Rao J, Goldman MP. Treatment of local, persistent cutaneous atrophy following corticosteroid injection with normal saline infiltration. Dermatol Surg. 2005;31(10):1340-1343. doi:10.1111 /j.1524-4725.2005.31216

8. Stassiy A, Khachemoune A. Unusual depressed areas on the arm and buttock. JAAPA. 2010;23(11):18. doi:10.1097/01720610201011000-00003

9. Ahmed I. Post-injection involutional lipoatrophy: Ultrastructural evidence for an activated macrophage phenotype and macrophage related involution of adipocytes. Am J Dermatopathol. 2006;28(4):334-337. doi:10.1097/00000372-200608000-00008

10. Oikarinen A, Haapasaari KM, Sutinen $M$, Tasanen K. The molecular basis of glucocorticoid-induced skin atrophy: topical glucocorticoid apparently decreases both collagen synthesis and the corresponding collagen mRNA level in human skin in vivo. $B r$ J Dermatol. 1998;139(6):1106-1110. doi:10.1046/j.1365-2133.1998.02646.x

11. Shiffman MA. Letter: treatment of local, persistent cutaneous atrophy after corticosteroid injection with normal saline infiltration Dermato/ Surg. 2010;36(3):436. https://doi. org/10.1111/j.1524-4725.2009.01468.x

12. Papadopoulos PJ, Edison JD. The clinical picture - soft tissue atrophy after corticosteroid injection. Cleve Clin J Med. 2009;76(6):373-374. doi:10.3949/ ccjm.76a.08096 